\title{
Structures, environnement et basculement dans le jihadisme
}

\section{Luis Martinez}

\section{(2) OpenEdition \\ 1 Journals}

\section{Édition électronique}

URL : http://journals.openedition.org/conflits/10912

DOI : 10.4000/conflits. 10912

ISSN : $1777-5345$

Éditeur :

CCLS - Centre d'études sur les conflits lilberté et sécurité, L'Harmattan

\section{Édition imprimée}

Date de publication : 20 avril 2008

Pagination : 133-156

ISBN : 978-2-296-05252-9

ISSN : 1157-996X

Référence électronique

Luis Martinez, «Structures, environnement et basculement dans le jihadisme », Cultures \& Conflits [En ligne], 69 | printemps 2008, mis en ligne le 17 juin 2008, consulté le 30 mars 2021. URL : http:// journals.openedition.org/conflits/10912; DOI : https://doi.org/10.4000/conflits.10912 


\title{
Structures, environnement et basculement dans le jihadisme
}

\section{Luis MARTINEZ}

Luis Martinez est directeur de recherche au CERI-Sciences Po. Il est l'auteur de plusieurs ouvrages: La Guerre civile en Algérie, Paris, Karthala, 1997 ; The Libyan Paradox, Londres, Hurst, 2007 et The Enigma of Islamist Violence, Blom A., Bucaille L. (eds), Londres, Hurst, 2007.

\begin{abstract}
Comment expliquer l'attrait pour le courant jihadiste de certains jeunes $\checkmark$ musulmans en France ? Cet article montre comment se construit la justification de la radicalisation et les étapes qui rendent légitime le passage à l'acte violent. Le processus de justification du passage à l'acte violent - à l'attentat-suicide par exemple - s'inscrit dans un contexte qu'il faut comprendre : quelles sont « les structures sociales et organisationnelles qui peuvent promouvoir dans un moment donné, l'attentat-suicide ? 1 ». Aussi, l'analyse du basculement dans la violence doit-elle recontextualiser les engagements et les trajectoires individuelles, car l'environnement dans lequel se construit le processus de justification apparaît comme déterminant. Le basculement dans la violence n'est pas le produit d'une frustration ou d'un symptôme psychologique. L'attentat-suicide, par exemple, est un véritable instrument de guerre. Il a un sens, répond à une logique et s'inscrit dans une finalité : un territoire à libérer, une communauté à reconquérir ${ }^{2}$. Les entretiens réalisés auprès de jeunes musulmans d'origine nord-africaine 3 permettent ici de souligner comment se construit la justification du passage à l'acte violent; ils permettent aussi de comprendre que, pour que l'acte violent se réalise, des conditions générales et structurelles sont nécessaires, sans quoi la justification du passage reste de l'ordre du discours ${ }^{4}$.
\end{abstract}

1. Guittet E.-P., "Les missions suicidaires, entre violence politique et don de soi ?", Cultures $\&$ Conflits, ${ }^{\circ} 63,2006$, pp. 171-174.

2. Blom A., Bucaille L., Martinez L. (eds.), The Enigma of Islamist Violence, Londres, Hurst, 2007.

3. Dans le cadre d'une étude sur le radicalisme, dix entretiens ont été réalisés en région parisienne en 2007. Nous remercions Kamel Cheklat et Hatim Ben Maimoun pour leur aide précieuse.

4. NdR : dans les différents extraits d'entretiens retranscrits ici, nous avons choisi de conserver les erreurs de langue et de syntaxe dans le souci d'en conserver l'authenticité. 
L'analyse des entretiens montre comment se structurent les principes de base du recrutement. Les étapes de la radicalisation sont les suivantes : la prise de conscience d'un monde musulman assiégé et agressé par l'Occident, la découverte du drame qui frappe les civils musulmans, et enfin le devoir de jihad afin de leur venir en aide. Cette aide peut prendre deux formes : le recours soit au jihad défensif en partant en Irak ou en Afghanistan soit au jihad offensif, en acceptant de frapper ici même l'agresseur, en l'occurrence un pays occidental. Il reste à aider les candidats potentiels à surmonter l'interdit de tuer des civils. L'environnement dans lequel ils évoluent met à leur disposition une infrastructure permettant de solutionner un certain nombre d'inquiétudes. Bien évidemment, chaque candidat potentiel appréhende le basculement dans la violence à partir de ses connaissances et de son expérience. Pour les vétérans de la guerre civile algérienne (1991-1999) 5 , « exilés » en région parisienne, le débat sur les attentats-suicides et le terrorisme les renvoie à leur passé :

«En plus des morts, les victimes civiles... derrière tout ça, il y a une idéologie dangereuse qui autorise à tuer des civils. Le plus choquant, quand on parle avec un terroriste... c'est avec conviction qu'il affirme qu'il a le droit de faire ça, alors qu'il est loin de la religion. On ne peut régler le problème idéologique et religieux par un discours politique... Tu ne m'as pas compris. Ce que je veux dire est que le fait de poser une bombe est un problème idéologique et religieux. Ce dernier ne peut être réglé politiquement. On peut le régler par une idéologie correcte... C'est la religion musulmane, celle que j’ai apprise en Algérie. »

\section{Les principes de base du recrutement jihadiste}

Internet est un formidable outil de propagation des principes de base du recrutement jihadiste. Les organisations jihadistes développent sur la toile les modules de prêt-à-penser qui nourrissent les candidats potentiels. Pour Denis Pluchinsky, «les principes de base du marketing jihadiste » sont les suivants : "l'islam est attaqué par l'Occident avec la complicité des dirigeants musulmans apostats; des femmes et des enfants musulmans sont violés et tués; c'est leur devoir en tant que musulman de faire quelque chose et de participer au jihad militant». Il souligne également: «en rendant disponibles des représentations visuelles, sonores et graphiques de l'information sur un réseau mondial et ouvert, Internet devient un moyen efficace pour les jihadistes de faire connaître et populariser leurs revendications $» 6$.

5. Martinez L., La Guerre civile en Algérie, Paris, Karthala, 2000.

6. Pluchinsky D., Le Journal hebdomadaire, interview, 21-27 avril 2007. 


\section{Les étapes de la justification de la radicalisation}

\section{L'islam est attaqué par l'Occident et en particulier par les Etats-Unis}

Quels sont les facteurs qui expliquent l'engagement de certains jeunes dans des réseaux islamistes susceptibles de faire basculer des individus dans la violence extrême ? Parmi les nombreux facteurs, l'un apparaît comme fondamental : l'image d'un monde musulman agressé par les Etats-Unis. Le sentiment que le monde musulman est vulnérable et qu'il faut le défendre apparait comme fondateur de l'engagement. Ainsi, tous nos interlocuteurs évoquent la nécessité de résister à l'agression. Ils invoquent un jihad défensif pour justifier les mouvements jihadistes :

«Le jihad de défense n'a pas besoin de fatwa, il est un devoir individuel pour chaque individu, c'est une manière de se prémunir contre les injustices... Un commun des mortels qui viendra t'agresser sans raison, dans ce cas, vous êtes en face d'un cas de légitime défense... Appelez-le comme vous voulez parce que cet individu voulait vous atteindre dans votre intégrité... Vous voulez dire à cet individu, ne vous défendez pas... Il faut lui reconnaître son courage, mais dans le cadre d'un jibad de défense et non offensif. On n'est pas dans le cas d'un jibad offensif. Il faut une structure, des conditions et des fatwas. Ce qui arrive en Palestine, en Irak, en Afghanistan, c'est un jihad défensif. Pourquoi les Français ont résisté à l'occupation allemande ? La résistance est un droit légitime, chaque peuple souscrit à cette logique. La religion garantit cela, la loi, le droit international... C'est un droit acquis qui n'a pas besoin de cadre juridique. »

Le jihad apparaît comme une réaction à la politique étrangère des EtatsUnis. Le lien est évident pour les personnes interviewées :

«Le terrorisme est une marque de fabrique américaine. Et si on essaye de le définir ? Il n'a pas de définition claire... c'est quoi le terrorisme ? Le fait de tuer un individu sans défense est un acte terroriste. Le terrorisme d'Etat est plus dangereux, l'Amérique est un Etat terroriste qui a envahi un Etat faible sans aucune forme de légitimité [el Haq].

Vous me dites ce qui s'est vraiment passé le 11 septembre... c'est une suite logique de beaucoup d'accumulations. En 1986, les Américains ont attaqué la Libye... Comment vous appelez ça ? Dans les années 1990, ces Américains ont envahi le Soudan, la Somalie, l'Afghanistan... Ce n'est pas du terrorisme ça ? Les Etats-Unis d'Amérique se sont constitués en Etat indépendant sur la base du 
génocide des peuples. Un individu qui n’a pas d'histoire [un passé] peut-il prétendre à un avenir ? L'histoire des Etats qui se revendiquent de la démocratie a un passé noir avec les Etats du tiers-monde. Les Etats-Unis sont le seul pays au monde qui a usé de la bombe atomique à deux reprises. Je veux comprendre : en quoi consiste le licite et l'illicite [el harem ona el hallal] chez eux ? La bombe atomique ? Les armes de destruction massive ? Soubhan Allah, louange à Dieu! Les Américains ont bombardé à deux reprises les Japonais. Le 11 septembre a été à l'origine de la mort de 3000 Américains. Combien de guerres ces mêmes Américains ont-ils mené ? Le Vietnam, le Japon, l'Irak, les Indiens... Voulaient-ils exterminer le monde entier ? Pour eux, il s'agit d'un conflit pour la survie, de vie ou de mort. Les Américains n’ont pas vécu 50 ans sans faire de guerre... Ils sortent d'une guerre pour en déclencher une autre. L'Amérique a entamé sa politique avec une guerre civile interne, au lieu d'orienter sa force intérieure vers le reste du monde. »

Face à la politique étrangère des Etats-Unis, le jihad semble donc s'imposer. La construction de la perception de la politique étrangère des Etats-Unis est importante car elle permet de justifier la violence réactive des groupes jihadistes. Alors que la diplomatie américaine insiste pour expliquer qu'il n'y a pas de lien entre la violence des islamistes à son encontre et sa politique étrangère, les individus qui intègrent les réseaux jihadistes le font avec la conviction que les Etats-Unis sont responsables des malheurs du monde musulman. Ils considèrent en outre que le gouvernement n'est pas le seul responsable de la politique étrangère, le peuple y joue aussi un rôle :

"Chaque individu a sa part de responsabilité dans ce qui se passe actuellement dans le monde. Qui a fait élire le président des EtatsUnis ? Ce peuple... pourquoi a-t-il voté pour Bush ? Ils l'ont élu pour un programme politique et ce peuple a pris connaissance de la politique de Bush. Vous pouvez me dire que le peuple américain est innocent!

Si l'Etat américain a envahi l'Irak, le peuple endosse l'entière responsabilité parce qu'il a opté pour ce programme... »

Une telle affirmation pourrait laisser penser que la violence contre les citoyens américains semble légitime aux yeux de ses personnes. Au contraire, la violence contre les civils est en réalité désapprouvée. Seul le jihad défensif est soutenu et encouragé :

«De mon point de vue, je fais la distinction entre organisations et actes terroristes... Tous les actes ne sont pas terroristes, je récuse les actes terroristes contre les civils. Je comprends les actes de résistance 
en Afghanistan... Ils sont les combattants de la liberté... ils ne sont pas partis aux Etats-Unis pour combattre le terrorisme. J'ai de la sympathie pour les gens qui combattent en Afghanistan, en Irak, en Palestine, au Soudan... [Un long silence] Il y a un point, celui qui fait un acte dans un métro, un train, un avion... Je ne suis pas d'accord. Les vrais moudjabidin sont ceux qui combattent le terrorisme américain et les sionistes. »

Dans cette perspective, le basculement dans la violence ne peut se faire qu'à travers le voyage vers un pays agressé, à l'instar de l'Irak ou de l'Afghanistan. L'idée de tuer des civils en Europe, ou en France, n'est pas présente. La vie quotidienne démontre que ces derniers ne peuvent être tenus pour responsables des "massacres » qui sont perpétrés contre les civils irakiens ou afghans. En revanche, partir faire le jihad est attractif. Mais, après l'expérience de jihad en Irak ou en Afghanistan, le même interdit - tuer des civils en Europe - se maintient-il ? Car la conviction de devoir partir faire le jihad est construite sur les images que véhiculent les médias qui couvrent les guerres d'Irak et d'Afghanistan. Mais, une fois confronté à la cruelle réalité de ces guerres puis rentré (au pays, en France...), il semble que l'interdit de tuer des civils en Europe puisse se diluer dans la conviction que ces civils doivent, eux aussi, payer le prix.

\section{Des civils musulmans sont tués dans l'indifférence}

L'information qui est véhiculée concernant la communauté musulmane agressée par l'Occident se greffe sur des faits historiques ou des événements avérés. Au sentiment d'injustice, doit s'ajouter celui de la révolte face à la violence. Dans ce cas, les jeunes interrogés basculent dans le sentiment d'amertume, ils éprouvent de la peine, de la pitié, une envie de vengeance. Le sort des Palestiniens revient en force pour pointer l'indifférence du monde occidental:

«Evidemment, ce qui se passe en Palestine et ce qui s'est passé pendant les croisades où 80000 musulmans ont été massacrés au nom de la religion et sans aucune forme de droit [...]. On ne peut pas justifier le massacre de 80000 musulmans sans raison valable. »

Le basculement s'opère lorsque le candidat potentiel éprouve un réel sentiment face aux malheurs de ses frères; il voit et vit le drame par procuration, et ressent de l'empathie pour eux :

"C'est vrai qu'il reste toujours difficile de voir des gens de sa communauté se faire maltraiter ou se faire spolier ou tout autre chose. Voir justement les gens de sa communauté se faire tuer.... enfin ça dépend de quelle région du monde... Se faire exclure de son pays ou se faire, comment dire, coloniser parce que, entre parenthèses, on dit que les 
colonisations sont terminées mais bon, se faire envahir par d'autres pays, c'est toujours triste et moi je me sens affecté, dans certains cas où des problèmes sont plus politiques, territoriaux ou religieux. Pour moi, la Palestine ce n'est pas un problème religieux, c'est un problème territorial et politique... C'est une histoire de terre qu'on a prise à certaines personnes et qu'on ne veut pas rendre, voilà, ça n'a rien à voir entre juifs et musulmans, moi c'est ma vision de la chose, il y a un côté sioniste qui veut le grand Etat d'Israël et, pour ça, il faut prendre toute la terre des Palestiniens et exclure les Palestiniens. »

Imprégné du sort de ses « Frères », le candidat potentiel considère qu’il est de son devoir de réagir. Au nom de l'islam, il doit participer à la protection de sa communauté :

«La résistance en elle-même, elle est légitime, la résistance... Quand on est opprimé, on doit bien résister, comme un musulman qui demain se voit opprimé par quelqu'un, il doit résister, et ne doit pas baisser les bras, maintenant les moyens qui y amènent... quelqu'un qui prend des armes pour défendre son bout de terre et sa maison, moi je trouve ça légitime, maintenant... Il y a des façons de le faire, on a affaire soit à une armée, soit à autre chose, donc en tant qu'armée, on se bat en tant que soldat. »

\section{Le jihad : un devoir}

Une fois convaincu que sa communauté est agressée par l'Occident, que ses «Frères » sont meurtris dans l'indifférence, le nouveau jihadiste est fin prêt à reconnaître la légitimité de la violence. Une organisation comme celle d'Al-Qaïda lui paraît un modèle de guerre par excellence :

«C'est mon point de vue, la force et la légitimité d'Al-Qaïda, c'est de combattre l'impérialisme. La guerre contre les Etats-Unis est une forme de légitimité... Ce sont les combattants de la foi. D’un point de vue religieux, selon le fikh [jurisprudence musulmane], si un ennemi envahit la terre musulmane, le jihad est une obligation individuelle. »

Le jih ad apparaît comme une forme d'auto-défense, un réflexe de survie. Pour le nouveau jihadiste, cette réaction est naturelle, elle est partagée par d'autres sociétés. Il reste à trouver un pays à défendre. Lequel choisir ? Comment faire ? Accomplir le jihad défensif signifie qu'il faut partir de France vers un pays agressé. Parfois, le doute s’installe quant au choix de destination, mais la conviction selon laquelle il faut défendre un territoire revient aussitôt :

«Ce serait mieux de me fixer des Etats... l'Irak, la Palestine, la Tchétchénie, l’Afghanistan... [Un long silence]. Comment dire... 
[Silence]... Un étranger qui occupe une terre... même en Tchétchénie, ce sont des séparatistes... Dans la Constitution américaine... un article parle de ça, de la défense nationale... Si l'intérêt national ou le territoire national est menacé par une force armée étrangère. C'est-à-dire, ce peuple a le droit de constituer une force armée pour défendre son territoire. Oui, c'est de l'auto-défense. Je soutiens les résistances légitimes contre une armée officielle occupante. »

Une fois assuré qu'il est prêt à combattre, bien qu'il ne sache pas encore où, à quels types de missions le nouveau jihadiste va-t-il se retrouver confronté ? Lesquels est-il prêt à effectuer ? Souhaite-t-il partir en Irak et participer à un jihad défensif ou bien est-il prêt à tuer des civils en Europe dans le cadre d'un attentat-suicide? Pour le nouveau jihadiste, c'est un véritable dilemme. Il est déchiré entre les appels d'Al-Qaïda à commettre des attentatssuicides en Occident et l'interdit de l'islam traditionnel de tuer des civils. Pour l'instant l'attentat-suicide n'est pas intégré dans le jihad. Celui-ci ne peut être réalisé que dans un pays agressé, l’Irak en particulier.

«Le jihad est ce qui est stipulé dans le Coran et la sunna. Si on parle du deuxième cas de figure, le cas du jihad entre un musulman et un mécréant, là, il y a plusieurs questions. La première, par quel droit un certain musulman va combattre un certain mécréant dans son propre pays $[\ldots . .$.$] ? Je considère cela comme un acte illicite qui dépasse la$ dignité humaine et je n'ai jamais entendu parler de ces actes à l'époque du Prophète. Pour le deuxième cas, le jihad est destiné à être exercé contre un vrai ennemi qui vient de l'extérieur pour coloniser le pays... ça, c'est un peu spécial et le jihad trouve sa vraie légitimité, comme le cas de l'invasion actuelle de l'Irak. Pour les musulmans, ils doivent combattre leurs ennemis juste dans leurs pays et non pas aller aux Etats-Unis et faire exploser les immeubles, des supermarchés, des femmes, des enfants et des vieux qui n'ont aucune appartenance ou relation avec le pouvoir... c'est-à-dire, les innocents. »

Et pourtant certains jihadistes basculent dans l'attentat-suicide. Comment l'expliquer ? Comment peut-on justifier des attentats-suicides au nom du jihad?

\section{L'attentat-suicide fait-il partie $d u$ jihad?}

Pour les personnes interviewées, l'attentat-suicide contre des civils en Europe n'est pas légitime.

«Je ne suis pas d'accord, je n'arrive pas à comprendre ce qui s'est passé à Casablanca, à Madrid et à Saint-Michel... [Silence]... En tant que musulman, je ne peux gober cela, l'islam m'interdit de tuer des civils et des non-combattants... » 
Pour les nouveaux jihadistes partisans du jihad défensif, l'attentat-suicide n'a pas de place dans leur engagement. C'est un interdit religieux qu'ils ne veulent transgresser, même s'ils comprennent que certains le fassent. Mais pour eux, ce serait se condamner religieusement à «l'errance»:

«Il y a une déférence [ikbtilaf] entre ceux qui usent du jihad pour le droit $[e l \mathrm{Haq}]$, c'est de la lumière et Dieu éclaire leurs chemins. D'autres estiment qu'ils font du jihad mais, en vérité, ils commettent des péchés et des massacres au nom de Dieu... Tuer des gens sans aucune forme de justice est une atteinte grave à leurs honneurs et biens. Dans la sourate El kahf, "ceux qui dans la vie commettent des pêchés et qui pensent bien faire... Je veux dire des gens dans l'errance". »

Les partisans du jibad défensif conçoivent leur engagement dans des combats sur le modèle classique des guérillas. La mort n'est pas valorisée, il faut combattre non pour mourir, mais pour vaincre son adversaire :

«Maintenant en guerre sainte, comment ça se passait à l'époque des Sahabas [compagnons du Prophète], moi ce que j'ai pu lire... je ne connais pas tout... On allait au combat en se disant je vais combattre... On ne dit pas je vais mourir aujourd'hui, si je meurs aujourd'hui el Hamdoulil Allah, si je rentre Al Hamdoulil Allab c'est comme ça que je pars... si tu es mort... tu es mort, Allah a décidé comme ça, c'est ça ton devoir, si tu te mets des bombes tu cours et tu t'exploses au milieu d'un camp, par exemple... des militaires, moi ça non. Je vois pas comme ça... détruire des infrastructure à l'explosif, comme ça se faisait à l'époque avec les Allemands, on faisait les trains ou des choses comme ça, s'attaquer à des militaires avec des mortiers ou des lance-roquettes, c'est légitime, ça c'est une guerre... c'est légitime... chacun défend son bien, maintenant l'attentat-suicide, ce n'est pas légitime. Et ceux qui... au nom de cette guerre sainte tuent ces civils... il y a des gens qui ne sont pas dans l'armée... je veux dire l'armée officielle et formelle et tout cela, mais puisqu'ils occupent nos terres ils ont le statut des guerriers. Il faut que ça soit bien défini, le civil. Le civil c'est celui qui n'a pas d'arme, il faut le prévenir, il faut lui dire que tu es dans un territoire dangereux... je sais plus quoi, mais le reportage où on parle de civils qui sont engagés par l'armée américaine pour $\mathrm{X}$ chose, pour des renseignements ou des machins comme ça, à mon avis, moi, ils sont des militaires, même s'ils n'ont pas de grade même si... Donc ils utilisent des civils pour des interrogatoires, ou pour des renseignements, ce sont des gens qui font partie de l'armée; maintenant la personne qui vient en Irak, un ingénieur qui travaille dans le pétrole, kidnappé ou on lui coupe la tête... non. » 
Mais les partisans du jihad défensif considèrent que les tueries de civils de chiites en Irak sont en revanche parfois légitimes, dans la mesure où ces derniers s'allient avec l'ennemi, les Etats-Unis :

« Ce qui se passe en Irak est qu'il existe des chiites et des sunnites. Les sunnites considèrent que les chiites sont des mécréants [kouffar]... selon l'adage qui dit "le chiisme est une forme de mécréance [kouffr] mais pas tous les chiites sont des mécréants". La résistance sunnite a compris cet adage, elle a dit que du moment que les chiites sont dans la mécréance, donc il faut les tuer tous en plus de leur soutien à l'armée américaine [un long silence suivi de réflexion]... Les civils chiites ne soutiennent pas les Américains mais il y a les milices chiites qui soutiennent les Américains. Si on parle de géopolitique en Irak, on trouve que les chiites se trouvent au Sud de l'Irak et, dès les premiers jours de l'occupation, ils se sont ralliés aux Anglais. Qu'est ce qu'ils ont fait ces Anglais ? Ils ont transféré le pouvoir local aux dirigeants locaux chiites... Que reste-t-il des sunnites... ? Là, ils font des attentats-suicides contre les civils chiites à Bagdad. Contre les Américains en Irak, je dirais oui. Contre les milices qui soutiennent les alliés, je dirais encore oui, mais pas contre les civils quelle que soit leur confession. Ce qui se passe au Liban, c'est une guerre provoquée par les chiites pour l'expansion de leur influence. La guerre a été causée par les chiites du Hezbollah même si c'est légitime de défendre le territoire national. Les chiites peuvent faire face aux juifs...»

Mais la résistance du Hezbollab durant la guerre de juillet 2006 a considérablement amélioré l'image des chiites auprès des nouveaux jihadistes. La rhétorique guerrière du Hezbollah apparaît comme une opération de marketing visant à améliorer l'image des chiites profondément ternie auprès des sunnites, et surtout d'Al-Qaïda, après leur alliance avec les Etats-Unis en Irak :

«Contre la politique israélienne et contre l'Etat d'Israël. Je n'ai pas terminé avec le Liban. Les chiites ont démontré qu'ils peuvent faire face aux juifs. Ce qui leur a donné une bonne image dans le monde musulman. Ce que fait Ahmadinejad démontre un certain courage face aux Américains. Ces deux images au Liban et en Iran ont donné une bonne image du monde musulman. »

En revanche, pour les jihadistes, l'expansion chiite est perçue comme une menace qu'il faut détruire, surtout si elle concerne des régions à très grande majorité sunnite, comme l'Afrique du Nord :

«En Algérie, les chiites dans deux villes algériennes [Oran et Batna] observent les coutumes des chiites irakiens. Ces coutumes sont interdites par la sunna et le Coran dans un pays sunnite comme 
l'Algérie. Je vois un autre danger que l'islam politique... c'est le problème chiite. C’est le microbe chiite... [Un long silence]. En Algérie, actuellement, le problème politique était le terrorisme mais avec Al-Qaïda, c'est grave. Les groupes armés ont été adoptés par Al-Qaïda... et si on ajoute l'émergence des chiites en Algérie ! »

La situation est grave, dans la mesure où elle laisse à penser qu'une guerre entre les chiites et les partisans d'Al-Qaïda replongerait l'Algérie dans la spirale de la violence mais, cette fois-ci, pour des raisons confessionnelles.

Cependant, l'insistance concernant l'interdit de tuer des civils ne doit pas faire oublier, selon eux, que les principaux responsables de tueries de civils sont les Etats et non les organisations jihadistes qui ont basculé dans le jih ad offensif :

«Selon mon opinion, je ne suis pas au courant de ce qui se passe, ces Afghans ont été gérés et réprimés dans leurs pays respectifs. La violence de l'Etat engendre une contre-violence... regardez ce qui se passe en Arabie saoudite par exemple. En Algérie, au contraire, ce sont les militaires qui sont à l'origine de ces événements. L'Etat algérien a créé son propre FIS ${ }^{7}$ pour tuer les civils... ce n'est pas ça l'islam, celui qui tue quelqu'un avec un pacte [convention] ne verra jamais le paradis. Le terrorisme d'Etat, c'est l'Amérique qui le pratique. Les médias ne reflètent pas la vraie opinion des gens. »

La logique de recrutement et de basculement dans le jihad s'articule autour d'un discours faisant de l'islam une religion agressée par l'Occident, puis d'une mise en scène montrant les tueries de musulmans dans l'indifférence générale, pour conclure par la nécessité de faire le jihad afin de protéger ses frères en religion. Une fois convaincu du devoir de faire son jibad, le nouveau jihadiste se heurte au choix de sa destination (Irak, Afghanistan, Tchétchénie, Palestine etc.). Il est soumis au dilemme du jihad qu'il veut réaliser : offensif ou défensif ? Pour le moment, le jibad défensif est celui qui recueille le plus grand nombre de candidats, car il s'inscrit dans la lignée de l'islam traditionnel et proscrit le meurtre de civils. Au contraire, Al-Qaïda valorise le jihad offensif et encourage les attentats-suicides contre des civils en Occident. Nos interviewés, de jeunes musulmans en France, sont très clairement attirés par le jihad défensif et donc plus enclins à faire leur devoir en Irak plutôt qu'à chercher à mourir en martyr dans le cadre d'un attentat-suicide. Il reste à mesurer l'impact de l'expérience du jihad en Irak par exemple, sur ce genre de conviction. En somme, l'Irak peut représenter pour des partisans du jihad défensif l'école de guerre qui les amènera à basculer dans un jihad offen-

7. FIS : Front islamique du salut, parti vainqueur des élections municipales de 1989 et législatives de 1991 en Algérie. A la suite de l'interruption du processus électoral par l'armée, l’Algérie entre en guerre civile (1991-1999). 
sif, c'est-à-dire à accepter ce qu'ils refusent pour l'instant, de mourir dans le cadre d'un attentat-suicide. Pourquoi le jihad en Irak est-il à même de favoriser ce changement auprès des nouveaux jihadistes ? En France, leur croyance s'est construite, comme nous allons le voir, à partir de lecture de fatwas, de la mosquée, de la télévision, etc. En Irak, il est fort à craindre qu'ils seraient sous l'emprise d'une information véhiculée par les réseaux d'Al-Qaïda. Finalement, l'environnement dans lequel ils évoluent en France produit des garde-fous qui limitent la propension à passer à l'acte, alors qu'en Irak, leur environnement favorise au contraire la promotion de l'attentat-suicide.

\section{Structures et environnement de la radicalisation}

Les nouveaux jihadistes partisans d'un jihad défensif évoluent dans un environnement où la mosquée, la lecture des fatwas, les médias et la famille constituent des structures importantes dans la construction de leur croyance. Aussi est-il possible de reconstruire leur environnement de façon schématique. Le processus de radicalisation des partisans du jihad défensif est long et jalonné d'étapes, ce qui le rend prévisible. Leur formation religieuse et intellectuelle, leur capacité à analyser les relations internationales permettent de placer les partisans du jihad défensif dans la catégorie des cadres du jihadisme beaucoup plus que dans celle des exécutants. Ils sont donc armés pour convaincre ou influencer d'éventuels exécutants. Ces derniers sont plus enclins à des réactions émotionnelles et donc à des comportements imprévisibles qui peuvent les amener à basculer dans la logique d'un attentat-suicide. Finalement, les personnes interviewées se situent dans des zones intermédiaires. Elles sont imprégnées du corpus religieux et intellectuel des jihadistes, elles vivent dans un environnement économique et social défavorisé et sont en contact avec des individus susceptibles, en raison de leur conversion aux radicalismes islamiques, de basculer de façon imprévisible dans la violence.

\section{La mosquée}

La mosquée constitue l'élément central dans l'environnement des partisans du jihadisme défensif. Les halaquates (cercles d'initiation à la religion) les socialisent et, dans le même temps, les confrontent à diverses interprétations. La mosquée permet de dialoguer sur l'actualité, d'aider les fidèles à définir ce que l'islam permet et interdit. C'est le lieu de rassemblement de diverses générations qui ne partagent pas toujours les mêmes inquiétudes. Aussi le rôle de l'imam est-il central :

«La communauté est indissociable de la mosquée car elle est le centre géométrique de notre vie. Pourquoi je dis cela? Au sein de la mosquée, on ne parle pas des affaires politiques car la communauté des anciens est très inculte politiquement. Elle ne pense qu'à son quotidien car, pour la majorité, elle vit avec un petit salaire. En ce 
qui concerne la deuxième génération, qui est elle très cultivée, elle parle politique en dehors de la mosquée, elle essaie de former un corps pour défendre ses intérêts, par exemple, l'affaire du voile, la guerre en Irak, le problème palestinien. Cependant, il peut y avoir une contradiction : le politique et le religieux sont inséparables en islam. Si à la mosquée, on ne parle pas politique, l'imam dans ses prêches et ses invocations, appelle à la prise de conscience des agressions politiques et des remises en cause des musulmans en général et de l'islam en particulier. Le militantisme musulman n'existe pas actuellement, mais il est en train de naître avec cette deuxième génération de musulmans. Certains musulmans, fervents pratiquants, faisant partie de l'élite et ayant conscience que la religion peut apporter un plus aux problèmes économiques et sociaux, s'inscrivent dans les partis politiques républicains. »

La mosquée offre les arguments religieux qui permettent de convaincre le fidèle de la justesse de son opinion. Face aux attentats-suicides, à la mort de civils et au jihad, de nombreuses interrogations sont formulées. Les prouesses des partisans d'Al-Qaïda sont admirées mais, dans le même temps, elles soulèvent des doutes sur la licéité de certains actes. La mosquée est le réceptacle de ces inquiétudes :

«Je suis contre le terrorisme, contre le fanatisme et contre le fait d'utiliser Allah comme prétexte pour tout faire. La voie pacifique, le dialogue entre les différentes communautés religieuses aussi bien avec la République, sont un gage de sécurité effective et collective. Il faut éviter l'amalgame entre islam, islamisation et terrorisme. Les médias... je suis très en colère contre les médias... au lieu d'être un vecteur d'information objective, ils ont été les témoins et les acteurs. Ce détournement des esprits nourrit la haine, les suspicions envers la communauté musulmane. Cette dernière, depuis le 11 septembre, n'a cessé à chercher de reconstruire son identité pour montrer qu'elle est différente de ce qu'on a voulu faire croire d'elle. Il n'est pas évident pour un musulman barbu avec une tenue traditionnelle qu'il s'inscrit dans un espace privé et public en respectant les valeurs de sa communauté et de la République française... »

La mosquée est également un espace qui permet de clarifier, grâce à l'imam, des troubles profonds comme la question du meurtre politique en islam. Face au déchaînement de la violence dans la société musulmane, une interrogation est posée à propos des victimes : qui a-t-on le droit de tuer ?

«Ce n'est pas légitime, c'est intolérable parce que la religion islamique interdit de tuer un innocent. Un hadith, si tu veux, a interdit de tuer une âme croyante, une âme d'un mécréant dhimi, un mécréant 
pacté [mouaâhid] et un mécréant pacifiste [moussalem]. Un mécréant pacté [lié par un traité] veut dire... qu'il existe un traité entre deux Etats, musulman et mécréant [kafir]. [Un long silence] bon, je parle en arabe classique... Un mécréant dhimi veut dire un mécréant qui vit dans un Etat musulman et qu'il ne faut pas le tuer parce que le chef de l'Etat [el hakem] lui a autorisé d'y vivre et il se trouve sous sa protection où il ne faut pas désobéir à ce hakem en tuant ce mécréant. Le mécréant pacifiste est celui qui ne porte pas atteinte à l'honneur et aux biens des musulmans, c'est pourquoi il ne faut pas le tuer... Je l'ai entendu aujourd'hui dans la prière du vendredi dans une mosquée de la région parisienne. »

Les attentats du 11 septembre 2001 ont provoqué une suspicion généralisée sur les jeunes islamistes, en particulier sur les salafistes. Pour certains de ces derniers, il devient de plus en difficile de pratiquer leur religion dans un environnement politique et social très méfiant face à ce courant religieux. Très présent en Algérie, le courant salafiste connaît un fort développement parmi les jeunes musulmans en Europe. En Algérie, le salafisme se divise en trois courants ${ }^{8}$ : un "salafisme scientifique » qui oblige le peuple, selon la tradition, à obéir au Prince; un «salafisme actif » qui est l'œuvre des ex-radicaux du FIS. Ils œuvrent à faire de l'individu une "forteresse » bâtie sur des valeurs islamiques et, enfin, un «salafisme djihadiste » incarné par le GSPC ${ }^{9}$. Les deux premiers courants sont très largement majoritaires dans la mouvance salafiste. Ils apparaissent comme une réaction religieuse à la guerre civile : restaurer l'image et le message de l'islam après l'usage qui en a été fait par les groupes islamistes radicaux et rendre aux croyants musulmans algériens le chemin de la paix intérieure par la réappropriation des valeurs islamiques fondamentales. Ces deux courants sont encouragés par le régime, ils permettent le recyclage des valeurs de l'ex-FIS du champ politique vers l'individu. Le projet des courants salafistes est de promouvoir « l'individu-forteresse », le musulman animé par des valeurs islamiques, et non plus d'instaurer un Etat islamique. Ceci dit, pour le régime, le salafisme, y compris dans sa version la plus pacifiste, demeure une menace dans la mesure où ses «valeurs » et certaines de ses « pratiques » semblent incompatibles avec celles de «l'islam officiel ». Son contournement se fait au travers de petites subtilités qui n'en demeurent pas moins porteuses d'une symbolique forte, celle de la défiance envers le régime. Ce sont des hauts-parleurs qui, dans certaines mosquées, appellent à la rupture du jeûne « dix minutes avant l'beure légale », ou bien la diffusion de prières «taraouih », voire l'importation du Coran comportant, selon le ministère des Affaires religieuses, « des altérations graves et malveillantes des versets $d u$ Coran », etc. Le troisième courant est un défi sécuritaire pour le régime, c'est le plus médiatique car le plus violent. En Europe, et en

8. Le journal Magharebia, 22 décembre 2006.

9. Groupe salafiste pour la prédication et le combat. Le 27 janvier 2007, Le GSPC se rallie à $A l$ Qaïda, le GSPC change de nom et devient «l'Organisation Al-Qaïda au Maghreb islamique ». 
France en particulier, les salafistes pacifistes sont victimes du courant jihadiste affilié au GSPC.

« J'ai lu beaucoup de choses dans ce domaine en Algérie, mais il faut préciser une chose... j'ai lu ça du courant salafiste en Algérie. Le groupe salafiste armé n'a rien avoir avec ce courant... c'est tout à fait différent. Je lis des livres de théologiens de l'Arabie saoudite comme El Albani. Ce sont des gens qui comprennent mieux la religion, ça c'est du salafisme scientifique... et j'ai beaucoup à dire sur ce sujet. »

La mosquée est l'espace où se côtoient de jeunes partisans du jihad défensif, prêts à partir faire le voyage en Irak, des salafistes, des musulmans pieux... Chacun connaît chez l'autre son périmètre d'action. Comment être un «bon » musulman ? Faut-il partir en Irak pour défendre ses « frères » en religion ? Doit-on les venger ici ? Ou bien ne serait-il pas mieux de répandre le vrai islam auprès de ses «frères égarés » influencés par des médias, qui diffusent une image erronée de l'islam ? La mosquée, c'est la cohabitation d'un ensemble de questionnements. Aussi pour certains, face à la prolifération des interprétations, un retour aux sources devient impératif :

«Pour moi, être un bon musulman, c'est suivre el Kitab [le Coran] et la sunna, ce sont les deux choses essentielles pour nous guider dans l'islam, pour moi la base de l'islam, c'est le monothéisme, on est tous d'accord là-dessus, mais la différence entre nous et les autres religions du livre c'est annabi [le Prophète] avec sa Sunna Wa Kitab Allab [Coran], qui est la parole d'Allah soubhanabo Wa ta'la [louange à Dieu], donc on doit se référer à cela à chaque moment de notre vie, à chaque acte qu'on doit faire, et chaque problème on doit essayer, on doit essayer de trouver... avec les gens qui ont de la science, ou en faisant Salat Al astikhara [une prière de nuit où l'on se remet complètement à Dieu], et moi c'est comme ça que je vois l'Islam bi Idhni Allah. »

Sur ces divergences idéologiques se greffent des appartenances nationalistes qui mettent à mal le sentiment communautaire d'appartenir à l'Oumma. L’imam, là aussi, rappelle les principes de base du musulman :

«En théorie elle existe, parce qu'on est musulman et tout musulman doit faire partie de la même communauté, après, moi, j'essaie de m'intégrer dans cette communauté, j'essaie de me faire accepter, ce n'est pas toujours facile. Dans tes questions, il y a déjà les prémices du problème de l'Oumma parce qu'il y a le nationalisme. L'imam d'Epinay ce vendredi parlait de cela en disant qu'on est tous des musulmans, qu'il n'y a pas d'Algériens, qu'il n'y a pas de Marocains, il n'y a pas plusieurs islams, il n'y a qu'un seul islam, et on fait tous partie de cet islam, à deux ou trois choses près. Des fois des... bon, 
en restant en règle générale normalement, il y a une Oumma et moi j'estime que mon frère est jusqu'à preuve du contraire, et même s'il me fait quelque chose, je n'ai pas à m'écarter de lui, c'est mon frère dans Dhin [frère de religion] et il fait partie de ma communauté... et je n'ai pas à entrer dans des jugements contre lui. »

Si la communauté est désunie, si elle ne parvient pas à définir la bonne conduite à suivre face à la guerre en Irak, aux attentats-suicides, aux meurtres de civils, certains croyants cherchent à s'affranchir de ces contradictions afin de vivre leur religion en symbiose avec Dieu :

« Je vis mon islam avec ferveur, avec foi, avec croyance, dans le sens de certitude d'un Dieu unique. J'ai besoin de cette foi pour vivre ma religion pleinement en symbiose avec la société dans laquelle je suis inscrit, je n'essaye pas d'imposer quoi que ce soit dans ma religion avec les personnes avec qui je vis, c'est-à-dire la famille, les amis, les personnes dans le monde professionnel, social et économique. Comment je vis la foi, je la vis comme un équilibre entre Dieu, moimême et les hommes en général, c'est-à-dire la société. »

Si la mosquée est un espace fondamental dans l'environnement des jeunes musulmans, la lecture des fatwas des grands théologiens est tout aussi fondamentale dans les étapes de l'engagement.

\section{Les fatwas et l'attentat-suicide}

Les fatwas permettent aux partisans du jihad d'élucider un certain nombres d'interrogations : est-il légitime de combattre un régime impie ? Les civils d'un régime impie sont-ils des victimes légitimes ? Les terroristes du 11 septembre sont-ils des martyrs ? Une des personnes interrogées répond :

"La fatwa stipule "Celui qui ne gouverne pas selon les prescriptions de Dieu, ceux là sont des mécréants”... [un verset coranique souvent utilisé par les jihadistes qui interprète le terme "yabkoumou” en arabe par "gouverner” alors que d'autres interprétations font référence non à une forme de gouvernement ou pouvoir politique mais à un jugement d'ordre judiciaire]. Celui qui soutient ce gouvernement impie l'est aussi, c'est pourquoi il faut le tuer. Tous ces biens, son sang et son honneur sont licites. C'est une fausse fatwa... la preuve, je vous cite ce hadith du prophète, Que le salut soit sur Lui [QSSL]... Après la mort du roi de l'Ethiopie [habacha], le prophète a observé la prière de l'absent et ce, malgré la non-gouvernance de ce roi selon les préceptes coraniques. C'est une réponse suffisante pour les excommunicateurs [taqufiris]...» 
Les fatwas les plus recherchées sont celles produites par des théologiens de renom. Ces derniers s'opposent à l'usage des attentats-suicides dans le terrorisme car ceux-ci constituent une rupture dans l'histoire de l'islam. En effet, en recherchant délibérément la mort, le «terroriste » provoque son suicide. Or, en islam, le suicide est condamné et nombreux sont les théologiens musulmans qui ne l'assimilent pas à un acte de martyre. Pour l'imam Mouhammad Nasiroudin Al-Albani :

«Toutes les missions suicides de notre époque sont des actes impunis qui doivent tous être considérés comme interdits [Haram]. Les missions suicides peuvent être de celles qui amènent celui qui les fait éternellement dans le feu ou le mettre parmi ceux qui ne résideront pas éternellement dans le feu... Mais voir ces missions suicides comme un moyen de se rapprocher d'Allah [acte d'adoration digne d'éloges] en se tuant aujourd'hui pour sa terre ou son pays alors nous disons non! Ces missions suicides ne sont pas islamiques! 10 »

Ce rejet des attentats-suicides s'inscrit dans une lecture traditionnelle de la violence en islam. Les théologiens ont élaboré, au cours de l'histoire, une réglementation très précise des conditions d'usage du jihad et l'attentat-suicide n'y avait, jusqu'à présent, pas sa place, premièrement car la mort délibérée est associée au suicide et non à la recherche de la défense de l'islam, deuxièmement car il existe une distinction claire entre les victimes combattantes et non combattantes. Pour le théologien Ibn Taymiyya (1263-1328) :

"Ceux qui, comme les femmes, les enfants, les prêtres, les vieillards, les aveugles, les invalides, etc. ne peuvent être considérés comme "résistants" ou "combattants", ne seront pas tués, selon l'avis généralement admis, à moins qu'ils n'aient effectivement combattu uniquement ceux qui nous combattent, car nous voulons faire triompher la religion de Dieu ${ }^{11}$.»

Les attentats-suicides qui frappent les victimes sont toutefois justifiés par certains théologiens à l'instar d'Al Qaradâwi sous le prétexte que « chaque citoyen juif ou citoyenne juive, en Israël, accomplit son service militaire, et est donc un soldat potentiel 12 ». Al Qaradâwi précise toutefois que plus que l'attentat-suicide, ce sont les intentions qui le motivent qui accordent le statut de martyr :

«Tout musulman qui atteste qu'il n'y a de Dieu que Dieu et que Muhammad est le Messager de Dieu, qui ne s'est pas rendu coupa-

10. D’origine albanaise, le théologien Al Albani est considéré comme un salafiste. Formé en Syrie, il a enseigné à l'université islamique de Médine. Voir : « Des attentats-suicides dans la balance de la loi islamique », http://www.sounah.free.fr/dawah_attentat_albani.htm

11. Ibn Taymiyya, dans Laoust H., Le Traité de Droit public d'Ibn Taymiyya, Beyrouth, Institut français de Damas, 1948, p. 28.

12. Cité par Saad R., "Weapons of the weak”, Al Abram Weekly Online, 13-19 décembre 2001. 
ble d'apostasie (en raillant un élément de la foi, en reniant une obligation, en considérant licite un interdit péremptoire ou en méprisant un point consensuel de la Shari'a) - s'il est tué dans la bataille qui oppose les musulmans aux juifs impies - est un martyr musulman à part entière. Toutes les dispositions concernant les martyrs sont prises pour lui : il n'est ni lavé ni enveloppé dans un linceul et est enterré avec ses vêtements dans lesquels il a été tué, et ce, afin que les traces de sang et les blessures témoignent en sa faveur le jour de la Résurrection. Quant à la question de savoir si son combat et sa mort sanglante sont dans le Sentier de Dieu [Sabîl Allab] ou non, cela revient à ses intentions, à ses motifs et à ses finalités, lesquels constituent en islam le critère d'évaluation de toutes les actions... L'effort de lutte [Jihâd] en islam n'est pas un acte matériel. Il s'agit plutôt d'un sacrifice de soi et d'un des plus grands cultes qui permettent de se rapprocher de Dieu. C'est pour cette raison que l'agrément de ce culte est conditionné par une entière abnégation de l'intention envers Dieu, ainsi que par une purification du cour de tout motif matériel comme la quête de la renommée, l'orgueil héröque, le fanatisme nationaliste ${ }^{13}$. »

Deux défis sont donc posés au potentiel kamikaze afin d'obtenir ce statut : il doit prouver que les raisons qui l'amènent à perpétrer son attentat sont pures et doit démontrer que, par cet acte, il œuvre à la seule défense de l'islam. En effet, le suicide est un péché en islam, passible du châtiment éternel. Les kamikazes du 11 septembre 2001 soulèvent un problème théologique épineux : sont-ils des terroristes ou des martyrs ? Pour le Grand Mufti d'Arabie saoudite, Sheikh Al Aziz Bin Abdallah, de tels actes étaient plus proches du suicide que du martyre :

«Je n'ai connaissance d'aucune disposition de la loi religieuse concernant le fait de se tuer au milieu des ennemis... Ceci ne fait pas partie du jihâd... Bien que le Coran autorise et demande même de tuer les ennemis, cela doit être fait d'une manière conforme à la Shari'a ${ }^{14}$. »

En fait, s’il est épineux de reconnaître la pureté des intentions des candidats au martyre, il est plus facile de justifier ce statut par la défense classique de l'islam. En effet, les organisations islamistes justifient leur usage de la violence par leur volonté de s'ériger en défenseur de la communauté musulmane agressée. De la Palestine à la Tchétchénie, de l’Irak au Cachemire, les organisations islamistes dénoncent la faillite des élites des Etats arabes et musulmans

13. Al Qaradawi, théologien égyptien, doyen du College of Shariah and Islamic Studies et directeur du Center for Sunna and Sirab à l'université du Qatar. "Le martyr et l'expiation des péchés », 2 mars 2003, http://www.islamophile.org/spip/article555.htm

14. El Sharq Al Awsat, 21 avril 2001. 
à venir en aide à leurs coreligionnaires. L'incapacité des régimes arabes et musulmans à protéger une communauté musulmane justifie le jih ad contre les gouvernants impies qui l'agressent : russes en Afghanistan et Tchétchénie, israéliens en territoires palestiniens, indiens au Cachemire et américains en Irak. Dans cette perspective, les «nouveaux martyrs » sont ceux qui osent se sacrifier pour la défense d'une communauté musulmane finalement réinventée. Comme le souligne Olivier Roy :

«Cette Oumma imaginaire peut s'exprimer dans des paradigmes historiques (Empire ottoman), des mythes politiques (califat) ou dans les catégories de l'islam traditionnel (dar ul harb, dar ul islam, pays de guerre, pays de l'islam) mais brouillées puisque cela ne correspond plus à un territoire ${ }^{15}$. »

En réalité, le problème ne se pose pas concernant la légitimité de la résistance (palestinienne, tchétchène), mais sur la licéité de l'attentat-suicide au regard des principes de l'islam. La raison principale est, bien sûr, normative : l'interdiction du suicide en islam (et la légitimité des attentats-suicides fait, pour cette raison, l'objet d'un intense et vif débat entre théologiens musulmans). L'acte est donc envisagé comme l'accomplissement, positif, de la volonté de Dieu; d'autant que le «martyre» n'est pas envisagé comme l'anéantissement de soi.

\section{Les médias, l'école et la famille}

Les médias, l'école et la famille jouent un rôle mineur dans l'environnement des jeunes jihadistes : «L'école, juste un peu... » dit l'un d'entre eux, la famille beaucoup plus. Aussi, c'est bien évidemment la télévision qui joue le rôle central de médiatisation:

«Avec tout ce qu'on entend de nos jours, c'est plutôt avec la télé qu'on est au courant de tout ça. Ce qui se passe, c'est plutôt au niveau médiatique. Maintenant, je ne peux pas vous dire exactement qu'estce qui se passe là-bas, parce que si je me réfère aux médias, pour moi c'est du faux et je n'y crois pas. Peut être qu'il y aura des vérités, 5$10 \%$ de vrai, mais le reste, est-ce que c'est vrai ? Maintenant, ça reste mes frères, mais la seule chose, le seul pouvoir qui me reste à faire aujourd'hui, c'est des dou'a [les invocations]. Mais ce qui se passe làbas, c'est triste. Ce qui se passe en Palestine, je trouve ça injuste, mais on ne peut rien y faire. Maintenant la seule chose qui nous reste comme pouvoir, c'est faire des dou'a, c'est la première des choses, et invoquer Allah. Et maintenant, si les musulmans le faisaient, norma-

15. Roy O., L'Islam mondialisé, Paris, Seuil, 2002, pp. 162-163. 
lement tout devrait être réglé. Mais si les choses devaient se passer comme ça, si les choses étaient dans le bien ou dans le mal, dans tous les cas, c'est un destin d'Allah. »

Selon les personnes interviewées, Internet représente le meilleur moyen d'accès aux fatwas, aux informations « fiables » sur l'Irak et l'Afghanistan.

\section{Le basculement vers l'attentat-suicide}

L'attentat-suicide soulève de nombreuses interrogations. Aller faire le jihad fait « rêver " certains, mais tuer des civils paraît encore insurmontable. Aussi beaucoup comprennent ceux qui le commettent sans pour autant se sentir eux, personnellement, disponibles pour l'accomplir.

\section{La comprébension de l'attentat-suicide ne signifie pas son approbation}

A la question: "Tu n'as jamais rêvé de faire le jihad? » la réponse de notre interlocuteur est claire :

«Bien sûr, oui. Tout croyant doit avoir la volonté et la détermination de le faire, après il faut avoir la science pour l'accomplir. Il faut suivre l'avis des savants qui sont bien trempés dans la religion. »

La perception d'une communauté musulmane agressée par l'Occident, et en particulier par les Etats-Unis, fait apparaître le jihad comme une nécessité. Mais l'engagement dans le jibad n'est pas seulement une affaire personnelle, il doit s'inscrire dans un cadre religieux qui le cautionne. Dès lors, la position des théologies est fondamentale selon la personne interrogée. Leur légitimité est indiscutable. Le problème se pose lorsqu'il s'agit de savoir si «les attentats-suicides font partie $d u$ jihad ». L'innovation d'Al-Qaïda est de chercher à légitimer, dans le concept de jihad, l'attentat-martyr. La controverse est générale au sein de la communauté musulmane. Pour notre interlocuteur, si le jihad est une nécessité, l'attentat-martyr pose un problème par le «carnage » qu'il provoque :

«Car il y a une grande confusion chez la majorité des gens lorsqu'on évoque ce terme. Il ne faut pas considérer que mes réponses sont vraies, parce que je reste ignorant. Et le problème, c'est que je suis en train de faire ni fatwa ni rien, je donne juste une opinion, mais qu'il ne faut pas prendre en considération à $100 \%$. C'est-à-dire que moi, je suis encore un chercheur; je cherche, j'apprends la religion. Je suis encore très jeune. Et maintenant, ce qui se passe dans le monde à ce sujet-là, moi je ne porte pas de jugement. A cette heure-ci, il n'y a rien, vraiment par ici, il n'y a pas de jihad, ni en Palestine, ni en Irak, ni ailleurs. Le jihad c'est partir au combat, pas dans l'esprit de se suicider, mais de revenir. A cette heure-ci, les gens qui sont là-bas, les pauvres, 
on peut les comprendre dans leur situation. Ils grandissent avec leur père mort, ils ont vu leur famille mourir... ou les Tchétchènes. On comprend leur situation. Pourquoi ils font ceci. Mais par rapport à notre religion, c'est erroné, c'est faux. Maintenant, aujourd'hui, moi je dis y a pas, si on n'est pas encore prêt. Parce que quand je regarde l'histoire, le Prophète (Paix soit sur Lui, PSL) a commencé par le Tawhid, il a commencé d'abord par faire adorer aux gens Allah. Ensuite par faire découvrir sa sunna, et au fur et à mesure, quand il a vu qu'ils étaient prêts pour se défendre, il les a appelé. Mais aujourd'hui, on n'est pas prêt. On n'est pas prêt en tant que musulman. Par exemple, si les musulmans avaient envie de faire quelque chose, de déclarer une guerre ou quoi que soit, ils ne sont pas prêts militairement ou religieusement. Maintenant, avec ce que l'on entend tous les jours, ça parle, ça parle, ça parle d'une bouche à l'autre, ça augmente, ça aggrave les choses. Aujourd'hui, chacun fait son travail, on se laisse faire, et ça c'est partout. C'est normal ce qui se passe aujourd'hui. Je le répète bien, ce n'est pas une fatwa, c'est rien, c'est bien ce que je veux faire comprendre. Mais faut bien garder à l'esprit que, quand on regarde dans l'histoire, les musulmans partaient en guerre, c'était pas pour tuer. Ils partaient juste pour occuper la religion. Eh bien, on va prendre l'exemple de l'Espagne. Quand ils sont partis conquérir l'Espagne, ils ne sont pas venus pour apporter la tuerie. Ils ont apporté la religion. Ils ont mis le pouvoir en place. Mais ils ont laissé les autres dans leur liberté de choix, mais ils vivaient sous l'empire musulman. »

Dès lors, l'interviewé comprend les motivations sociales qui provoquent les actions terroristes, mais ne les considère pas comme légitimes du point de vue religieux. A la question : "les attentats de Madrid, de Londres et de NewYork font-ils partie $d u$ jihad ? ", il répond :

«Ecoute-moi mon frère, Il n'y a pas pour le moment de jihad. Ce sont des actions de gens sincères dans leur religion, mais erronées dans leur compréhension et menées par leur haine et leur sentiment d'injustice. »

Sa désapprobation des attentats-suicides se fonde sur la mauvaise interprétation du jibad par les terroristes. Il ne condamne pas leurs actions, il éprouve de la compréhension mêlée d'un sentiment de pitié. Les kamikazes sont perçus comme des individus qui agissent par conviction : ils sont persuadés de faire le bien pour leur religion, alors même qu'ils la desservent.

Cette approche de l'attentat-suicide apparaît comme la plus mesurée. Elle s'inscrit tout d'abord dans une reconnaissance des théologiens sur les affaires religieuses. Ces derniers ne sont pas perçus comme illégitimes car inféodés à des régimes politiques arabes alliés de «l'Occident ». Cela signifie que, pour eux, 
l'attentat-suicide peut devenir légitime si les théologiens le cautionnent, auquel cas l'attentat deviendrait un acte de martyre. Les terroristes qui pratiquent des attentats-suicides sont à leurs yeux parfaitement compréhensibles, car ils agissent sous l'emprise d'émotions (colère, sentiment d'injustice, haine) mais sont convaincus de se sacrifier pour le bien de leur religion. Aussi, ces personnes partagent-t-elles avec eux la même lecture politique (le jih ad est nécessaire), mais alors que les kamikazes agissent sous l'emprise des émotions, lui serait prêt à le faire à la seule condition que les théologiens, c'est-à-dire la raison islamique, définissent comme permise la tuerie de civils dans le cadre du jibad.

\section{L'attentat-suicide, un acte de résistance}

D'autres considèrent l'attentat-suicide comme légitime. Il n'invoque pas la nécessité d'une caution des théologiens. En réalité, les individus qui basculent dans le jihadisme sont l'objet d'une grâce de Dieu :

«Il y a celui qui obéit à la puissance de Dieu [moussakhar] et d'autres pas. C'est les attributs de Dieu. Qu'est-ce que je vous dis... il y a des personnes pieuses, bienfaitrices qui ont la faculté par la grâce de Dieu de protéger cette religion... Elles sont l'outil de Dieu et Dieu les a consacrées pour leur bien [masslaha] d'être au service de la religion. »

Les kamikazes apparaissent comme des individus porteurs de la grâce divine, choisis pour défendre la communauté des musulmans. Ils sont semblables à des « élus de Dieu » prêts à se sacrifier, ils sont dès lors les « chevaliers du Prophète ». Ils inspirent respect et admiration. Pour eux, il n'y a ni haine ni violence dans les propos. L'attentat-suicide est légitime dans le cadre de la résistance.

« Sincèrement, le passage à l'acte est une autre question. Je ne suis pas disponible pour le moment... [un large sourire] Je suis quelqu'un qui aime la paix, je suis pacifiste et j'aime l'humanité. Si un jour, je vois quelqu'un dans un pays non musulman, je préfère dialoguer avec lui... Cette même logique, je ne peux pas l'appliquer en Afghanistan, en Irak et dans les pays victimes de l'invasion et du terrorisme d'Etat des Etats-Unis... »

A la question: "Dites-nous, ce qui vous empêche, vous, de procéder de la sorte?", une autre personne interrogée répond :

« J'arrive... j'arrive! Mais moi, j'ai dit que c'est normal... je n'ai pas dit que ce n'est pas légitime! Alors, que faut-il pour un vrai musulman ? Moi, je ne vois qu'une seule solution... laquelle ? C'est la résistance. Cette dernière ne s'oppose jamais avec la volonté du peuple, le droit de vivre pour tout le monde, aimer et respecter les gens quelles que soient leurs appartenances culturelles, religieuses et raciales. Dans ce contexte, je vous donne un exemple sur la manière 
de résister. Il s'agit de l'Iran... qui montre au monde entier qu'il est toujours dans une posture pacifiste et toujours claire envers ses adversaires [l'Occident au sujet de son programme nucléaire]. »

La résistance apparaît comme centrale, soit elle s'inscrit dans le cadre du jihad, soit dans celui d'une attitude pacifiste. En somme, soit elle prend la forme de la résistance irakienne, soit celle de l'attitude iranienne...

Dans le cadre d'une occupation de type irakienne, l'attentat-suicide est légitime lorsqu'il s'applique à des contextes particuliers comme ceux justement de l'Irak mais aussi de l'Afghanistan. Mais il ne peut se généraliser car il heurte alors un interdit, celui de la mort de civils.

« Je ne suis pas d'accord d'aller tuer des civils dans un autre pays, n'importe quel pays. Lors des attaques du 11 septembre 2001, j'étais parmi les gens qui s'étaient opposés à cet acte terroriste et j'ai eu de gros problèmes avec les gens de chez nous au Maroc. On s'est divisé en deux parties suite à ces événements. J'étais parmi ceux qui ont combattu l'idée d'une action jihadiste [haraka jihadial]... Ecoutez, je ne me suis pas changé depuis le 11 septembre... et je ne changerai pas [lame attaghaiar] et je soutiens ceux qui usent de l'auto-défense [combattent] en Afghanistan. »

Pour ces personnes, la mort des civils apparaît comme un tabou difficile à transgresser. Le jihad est légitime face aux Etats-Unis et à ses alliés, mais il ne peut être un instrument de vengeance face à des civils désarmés. Les groupes armés qui mènent la résistance dans des pays islamiques occupés ne peuvent pas appliquer les mêmes méthodes dans des sociétés en paix. Les fatwas qui justifiaient l'attentat-suicide en Irak ou en Afghanistan doivent devenir caduques en Europe ou en Afrique du Nord :

«La djamaa [les jihadistes] qui se trouve en Irak et en Afghanistan, après avoir regagné l'Algérie, a toujours continué de pratiquer la même fatwa jihadiste. Ces jihadistes se sont basés sur une fatwa religieuse, légitimant de combattre une armée envahissante... c'est un fait légitime. Le problème est que, de retour au pays d'origine, ces jihadistes utilisent toujours la même fatwa comme s'il s'agit d'une guerre contre une armée envahissante. »

Face aux inquiétudes qui pèsent sur la communauté musulmane (Oumma), la seule certitude est de s'en remettre à Dieu : lui seul a le pouvoir de choisir ses serviteurs, mais aussi de protéger les siens.

«N'oubliez surtout pas que quand Dieu veut protéger une chose ou un bien, il lui crée ses propres causes et il met à sa disposition 
l'obéissance de l'univers et le matériel par la grâce et la force de Dieu [taskhir] comme des personnes, une tornade et un phénomène naturel pour protéger la maison de l'islam [el bey].

L'Oumma ne s'édifie pas à partir d'un individu [un long silence]. La définition de l'Oumma musulmane est une idée absolue qui transcende l'individu. L'individu se dilue dans la collectivité et le contraire n'est pas vrai. Je sens cette appartenance... l'Oumma existe de part le monde et ses chagrins sont vécus de la même façon. L'Oumma dont je parle n'est pas l'Etat, ce n'est pas la même définition. Chaque être humain éprouve de la colère quand vous portez atteinte aux slogans de cette Oumma. Je vous donne l'exemple des manifestations contre le Vatican dans les pays arabes où des drapeaux français, danois ont été brûlés... les politiques occidentaux se sont mis en colère contre ces procédés... Comment voulez-vous que je ne me mette pas en colère quand on touche à l'Oumma? Comment voulez-vous qu'ils aient de l'amour propre [jalousie] pour leurs pays et pas nous?»

Dans cette perspective, les musulmans doivent chercher les causes du problème et s'en remettre à la protection de Dieu.

«Avez-vous l'envie d'en découdre un jour et qui vous empêche de passer à l'acte ?

Je veux comprendre les causes et les effets quant à la demeure [il fait référence à la Kaaba dans une formule consacrée arabe], Dieu est là pour la protéger. [Il raconte] C'est l'année de l'éléphant où un roi s'apprêtait à démolir la Kaaba, Dieu a envoyé une nuée d'oiseaux... et enfin sauva la Kaaba de la démolition. Ce que je veux dire, la religion et l'Oumma islamique n'ont besoin de personne pour la défendre. Je vous donne un autre exemple, le fleuve de l'Euphrate ne peut être atteint si des chiens s'amusent dedans... Il est trop vaste pour que des chiens arriveront à le souiller !»

Dans la recherche des causes, il apparaît que l'absence d'Etat islamique est la raison majeure de la faiblesse de l'Oumma. La prolifération des Etatsnations dans le monde arabo-musulman a divisé et donc affaibli les musulmans. Le combat stratégique, dès lors, doit être de parvenir à l'instauration d'Etats islamiques :

«Le problème essentiel est l'absence de l'Etat islamique... [Dawla el Islamiya], si elle y est, elle se comportera avec la même logique. Selon Sayed Quotb, si la dawla Islamiya s'institue, un certain nombre de problématiques [massail] se décanteront et deviendront clai- 
res. L'absence de l'Etat islamique a créé une opacité. La vision est devenue sombre. Ces Etats colonialistes nous traitent avec répression, guerre et invasion et d'un côté elles sont liées par des conventions... Il ne faut pas envahir des Etats liés par des conventions et traités. Les Etats-Unis disposent de bases militaires et des conventions et en même temps, ces derniers déclarent la guerre au Soudan, l'Irak et l'Afghanistan. »

Dans cet idéal-type de jeune islamiste en France, l'attentat-suicide ne fascine pas. Il est perçu comme un instrument de guerre au service de groupes de résistants. Cet idéal-type s'en remet à Dieu pour solutionner les problèmes qu'il a créés. Ceci dit, son discours est imprégné d'un sentiment d'injustice face aux pays occidentaux et en particulier envers les Etats-Unis. Il n'y a pas de haine de l'Amérique, mais seulement le sentiment qu'il est normal de résister par les armes face à un pays qui colonise des pays musulmans, comme l'Irak ou l'Afghanistan. Mais pour cet idéal-type, "la colonisation » de pays musulmans est le résultat de l'absence d'Etat islamique. L'Oumma est faible et l'Occident en profite. Il faut donc œuvrer à l'instauration d'Etats islamiques afin d'édifier un rempart face aux agressions de l'Occident.

Partir faire le jihad est un « rêve », car il permet de réaliser le sentiment de devoir défendre la communauté musulmane. Les candidats potentiels ont confronté leurs inquiétudes et leurs doutes dans les mosquées, ils ont lu les fatwas qui répondent à leurs interrogations. Ils sont conscients que leur engagement n'est pas le fruit d'une réaction émotive, ils sont armés pour débattre et convaincre de la justesse de leurs arguments. Pour eux, le jihad défensif est le seul légitime : il faut combattre l'ennemi là ou il agresse la communauté, c'està-dire dans des pays comme l'Irak, l'Afghanistan, la Tchétchénie, la Palestine. Tuer des civils en Europe pour venger les civils musulmans « massacrés » n'est pas encore une option qu'ils considèrent comme autorisée. En effet, ils sont sensibles à ce que disent les théologiens, et ces derniers condamnent dans leur majorité l'attentat-suicide contre des civils. Les dirigeants d'Al-Qaïda ne sont pas encore parvenus à convaincre tous les jihadistes de la légitimité de l'attentat-suicide. Alors combien parmi les personnes interrogées partiront vraiment combattre afin de réaliser ce « rêve » ? Continueront-ils à rêver, seulement ? Il est difficile de le mesurer. En revanche, de leurs propres aveux, les « frères » partis réaliser leur « rêve » n'en avaient jamais parlé auparavant... 